\title{
Fast Fabrication Process of Microfluidic Devices Based on Cyclic Olefin Copolymer
}

\author{
Aymen Ben Azouz ${ }^{1,2}$, Stephen Murphy², Shadi Karazi ${ }^{2}$, Mercedes Vázquez ${ }^{1,2}$, And Dermot Brabazon ${ }^{1,2}$ \\ ${ }^{1}$ Irish Separation Science Cluster, National Centre for Sensor Research, Dublin City University, Dublin, \\ Ireland \\ ${ }^{2}$ Advanced Processing Technology Research Centre, School of Mechanical and Manufacturing Engineering, \\ Dublin City University, Dublin, Ireland
}

\begin{abstract}
A new low-cost process for fast fabrication of multilayer microfluidic devices using cyclic olefin copolymer film materials is presented. This novel process consists of the fabrication of microfluidic features by xurography, followed by multilayer lamination via cyclohexane vapor exposure. Exposure time to this solvent and compression time were optimized for bond tensile strength. A three-layer microfluidic chip capable of withstanding back pressures up to $23 \mathrm{MPa}$ was fabricated in less than an hour. The suitability of this fast prototyping method for fabrication of functional UV-transparent microfluidic devices was demonstrated by development and testing of a microfluidic mixer and preparation of a polymer monolithic column within the microfluidic channel.
\end{abstract}

Keywords Bonding; COC; Films; Microfluidic; Micromachining; Micro-mixer; Monolith; Solvent; Vapor; Xurogaphy.

\section{INTRODUCTION}

Microfluidic devices are nowadays widely used in chemical, environmental, and biomedical applications. Typically a few square centimeters in size, they integrate simple or complex 3D microfeatures to allow for suitable handling of fluid volumes on the order of a few microliters, nanoliters, or even picoliters. Their development has introduced a positive transformation within chemical and biological research due to their capability to perform rapid and high-throughput analysis with minimal consumption of sample and reagents [1].They may also incorporate several laboratory functions in a single platform [2].

Standard methods for fabrication of microfluidic devices involve the bonding of two polymer or glass layers after having embedded the desired microfeature structure on the surface of one of the layers. The most common techniques employed in the fabrication of microchannels and other microfeatures are hot embossing, injection molding, soft lithography, laser ablation, and micromachining [3]. Other techniques such as xurography and thermoforming are more popular for fast prototyping of centrifugal microfluidic platforms from polymeric films [4]. In fact, polymers have become a very competitive alternative to glass or silicon for fabrication of microfluidic devices since polymeric chips are more suitable for mass production, and thus, more appropriate for production of disposable devices at lower costs [5]. 
On the other hand, bonding of substrate layers to enclose the channels still remains the most critical and inconsistent step in microfluidic chip fabrication [6]. Substrate bonding is even more critical in the fabrication of multilayer microfluidic platforms since good adhesion between layers is essential for preserving the optimal alignment of the internal 3D microstructures [7, 8]. Thermal bonding is the most widely used and established bonding method [9]. It involves increasing the temperature to or above the glass-transition temperature ( Tg) of the material, which may cause deformation and collapse of the microfeatures, especially for low aspect ratio channels and thin substrates [10]. Other common bonding methods include adhesive bonding [11], plasma modification [12], ultrasonic welding [9], and solvent bonding [13]. Solvent bonding methods are usually employed for bonding layers of the same polymeric material. They can be applied at room temperature [13], thus minimizing the risk of channel deformation. Alternatively, exposure to solvent vapors forces the polymer chains to become mobile and diffuse at contact with the other polymeric layer to form relatively strong covalent bonds across the interface [9, 14], while preventing the polymer swelling typically observed when in direct contact with liquid solvents. Moreover, solvent vapor bonding has been recently proved to reduce surface roughness in channels fabricated in polymeric substrates by rapid prototyping techniques [15].

In this work, a new rapid prototyping method for fabrication of multilayer microfluidic devices from off-the-shelf cyclic olefin copolymer (COC) films is presented. This method is based on the fabrication of microchannels and sample reservoirs on COC films by xurography, followed by solvent vapor bonding of the resulting layers for channel enclosure. COC films were selected in this work due to their chemical resistance and high optical transmission, allowing the use of organic solvents and on-chip optical detection in separation science applications [16]. Optical sensing of the light fluoresced from or absorbed by a liquid sample passing through a microfluidic channel is one of the most used techniques for species detection in analytical chemistry. Such a photometric detection system consists in exposing a sample within a microfluidic channel to a monochromatic light and the use of a photodetector to measure the intensity of the light transmitted from or emitted by the sample [17]. As the light source and detector are usually located outside the transparent medium in these systems, knowledge of how this medium absorbs or transmits the light spectrum is of critical importance.

COC is one of the most used polymers in this area $[13,18]$ primarily due to its high transparency in the UV region, low auto-fluorescence over a wide spectral range, very high glass-transition temperature, optimal stiffness, and high stability against hydrolysis and chemical degradation $[15,19,20]$. Although solvent vapor bonding methods have been previously used for bonding of COC sheets [15, 18, 21], bonding of thinner COC films (thickness, $188 \mu \mathrm{m}$ ) is demonstrated here for the first time. In previous works, the bonding of COC layers with thickness ranging from $0.5 \mathrm{~mm}$ to $1.2 \mathrm{~mm}$ following exposure to cyclohexane vapors was reported $[15,18,21]$. The whole two-layer chip fabrication process, including the fabrication of the microfluidic channels by micromilling [15] or injection molding $[18,21]$, required several hours. In comparison, a complete three-layer microfluidic chip can be fabricated in less than an hour by using this novel methodology. 
For validation of this new fabrication method, two different prototypes were tested: a microfluidic chip integrating a polymer monolithic column within the channel, and a Tshaped microfluidic mixer. The monolithic column was prepared in situ by UV-initiated polymerization taking advantage of the high optical transmission of COC films. Light transmission measurements showed no significant effect on the optical properties of the COC films following exposure to solvent vapors. Tensile tests and in-channel backpressure measurements were also performed in order to characterize the bond strength at the interface.

\section{EXPERIMENTAL}

\section{Microfluidic Chip Fabrication Process}

Microchannel and reservoir fabrication. COC films (ZeonorFilm ZF14, Zeon Chemicals Europe Ltd) with a thickness of $188 \mu \mathrm{m}$ were cut through via the xurography technique using a Craft Robo Pro S, CE5000-40-CRP, Graphtec Corporation, Japan. Channels and inlet/outlet reservoir layouts were first designed in AutoCAD (Autodesk, Inc.), see Figs. 1(a) and (b), and then imported into the cutting plotter's software (ROBO Master Pro). Xurography allowed the fabrication of microchannels and reservoirs in a just few seconds.

Solvent vapor bonding. After fabrication of the channels and inlet/outlet reservoirs, the COC films (thickness, $188 \mu \mathrm{m}$ ) were bonded by solvent vapor bonding. A procedure similar to those employed earlier for bonding COC sheets, which basically consisted of substrate exposure to cyclohexane $[15,18,21]$, was developed and optimized here for bonding of COC films.
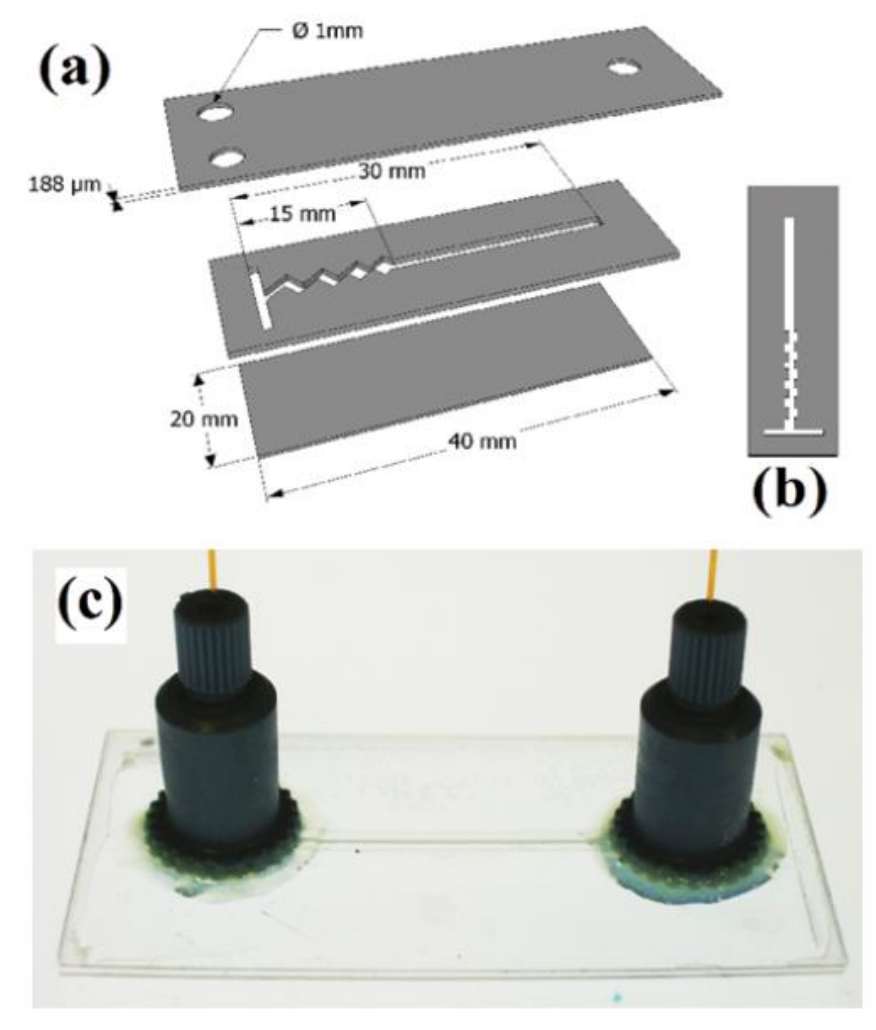

FIGURE 1.-(a) Schematic of the three-layer T-shaped mixer integrating a zigzag shaped microchannel and (b) the second layer integrated in a T-shaped mixer with obstacles on the channel wall. (c) Picture of a microfluidic device fabricated by xurography and solvent vapour bonding. 
Before bonding, the COC films were rinsed with isopropanol and deionized water, and then dried with nitrogen. In order to prevent any surface contamination, the films were carefully handled with tweezers while wearing gloves. Using a hotplate, the cyclohexane (LabScan Analytical Sciences) was heated up to $70^{\circ} \mathrm{C}$ to saturate a closed glass chamber with cyclohexane vapor. The glass chamber consisted of a Petri dish fitted with its corresponding lid. Once the glass chamber was saturated with cyclohexane vapor, the two COC films to be bonded were exposed to the vapor by fast swapping of the chamber lid with another lid that had the COC layers attached to its inner surface.

After exposure to the solvent vapor for a specific time, the two COC layers were immediately inserted into a customized in-house alignment jig, and brought into direct contact. For the purpose of the tensile test, half of the surface area of the samples was covered with a Teflon film to prevent bonding of the entire surface area. The alignment jig was then fitted into a pneumatic press and a constant pressure of $0.4 \mathrm{MPa}$ was applied for a set period. In a separate set of experiments, a vice was used for compression of the alignment jig containing the COC layers to bond and a constant pressure of $0.9 \mathrm{MPa}$ was applied for a set period. A load cell (RLC00500-RDP Electronics Ltd) was used to record the applied load, which was then divided by the COC bonded area to get the applied pressure. The bonded layers were then removed from the alignment jig and irradiated with UV light $\left(2 \mathrm{~J} / \mathrm{cm}^{2}\right.$ at 254 nm) using a Spectrolinker XL-1000 UV Crosslinker (Spectronics Corp., Westbury, NY, US) to increase the bond strength [18]. In order to connect the microfluidic chip to the macroworld via fused silica capillaries (id $100 \mu \mathrm{m}$ ), 6-32 coned NanoPort connectors (Upchurch Scientific) were finally fixed to the inlet/outlet reservoirs using epoxy glue. A picture of the resulting microfluidic chip is shown in Fig. 1(c).

\section{Tensile Testing}

Tensile testing of the bonded samples was performed using a Zwick-Roell $5 \mathrm{kN}$ tensile testing machine. A 6-mmlong section of the unbonded area between two bonded layers was gripped by the machine as shown in Fig. 2 .
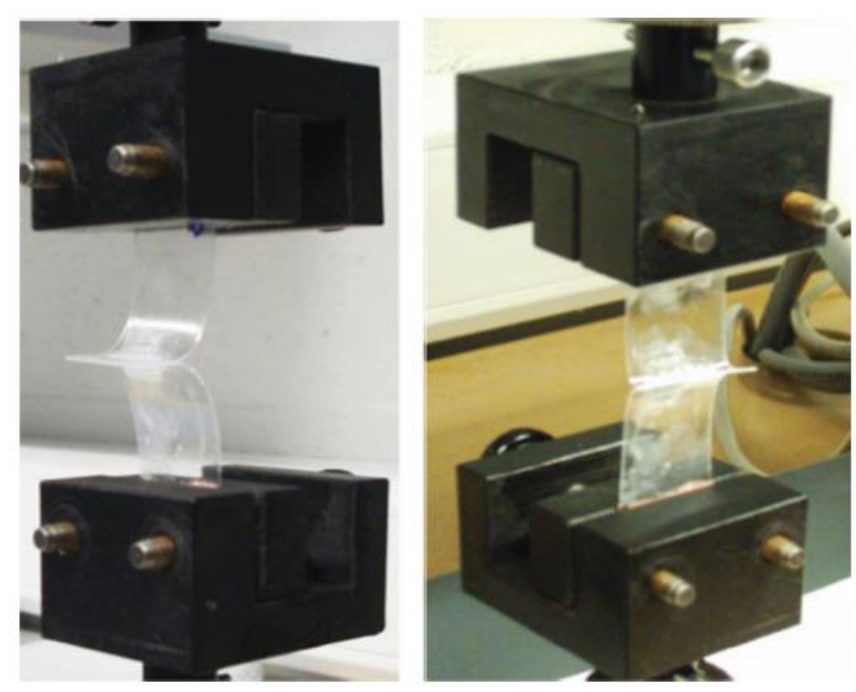

FIGURE 2 .-Picture of a bonded sample during a tensile test (left) and close to failure (right). 
The initial distance between grips was set at $20 \mathrm{~mm}$ and the rate of extension at 2.5 $\mathrm{mm} / \mathrm{min}$. Force vs. extension generated at the bonding interface was measured for each sample until bonding failure (i.e., full delamination of samples). Data from at least four tests per set of conditions were recorded. These data were then used to construct stress vs. strain curves, and to calculate the ultimate tensile strength (UTS).

\section{Back Pressure Measurements}

The back pressure generated within the microchannel of five different chips while pumping at increasing flow rates was measured using an isocratic HPLC pump equipped with a pressure sensor (Smartline Pump 100, Knauer). In order to easily detect any leaks, DI water containing a food color additive was pumped into the channel and visual inspection of the chip was also performed throughout the entire experiment.

\section{Optical Transmission Measurements}

An ultraviolet-visible spectroscope (UVMini-1240, Shimazdu) with a wavelength measurement range of 190- $800 \mathrm{~nm}$ was employed for light transmission measurements. The transmission spectra exhibited by COC films, $188 \mu \mathrm{m}$ thick, before and after exposure to cyclohexane, followed by UV irradiation and compression, were recorded.

\section{Micro-Mixers Fabrication}

In order to assess the feasibility of this novel fabrication method for fabrication of multilayer microfluidic devices, three different $\mathrm{T}$-shaped mixer prototypes were produced and their mixing efficiencies were compared. These three mixers designs fabricated were a classical T-shaped mixer, a T-shaped mixer with a zigzag shaped channel [22], Fig. 1(a), and a T-shaped mixer with additional obstacles on the wall [23], see Fig. 1(b). The micro-mixers consisted of three COC layers bonded by the solvent vapor bonding method presented in Section 2.1.2. The mixing efficiency for each of the three mixers was evaluated by mixing a solution of a $\mathrm{pH}$ indicator with an acid solution $(0.1 \mathrm{M} \mathrm{HCl})$ within the channel. Bromocresol purple (purple at $\mathrm{pH}>6.8$, yellow at $\mathrm{pH}<5.2$ ) and Bromothymol blue (blue at $\mathrm{pH}>7.6$, yellow at $\mathrm{pH}<6.0$ ) were used as $\mathrm{pH}$ indicators.

\section{Integration of a Porous Polymer Monolith Within the Microchannel}

A microfluidic device with one single channel (width, $600 \mu \mathrm{m}$; depth, $188 \mu \mathrm{m}$ ) (Fig. 1(c)) was used for the in situ preparation of a porous polymer monolith within the channel by UVinitiated polymerization. Benzophenone, butyl methacrylate (BMA), ethylene dimethacrylate (EDMA), 2,2-dimethoxy-2-phenylacetophenone (DMPA), and 1-decanol were all obtained from Sigma Aldrich (Dublin, Ireland) and used as received.

The channel was first washed with methanol and dried with nitrogen to remove any impurities resulting from the fabrication procedure. In order to promote the covalent attachment of the monolithic structure to the channel walls, those were grafted with a thin layer of BMA polymer following a two-step procedure described elsewhere [24]. 
Briefly, the channels were filled with a deoxygenated solution of $50 \mathrm{mg} / \mathrm{mL}$ benzophenone in methanol and irradiated with $2 \mathrm{~J} / \mathrm{cm}^{2} \mathrm{UV}$ energy at $254 \mathrm{~nm}$. The channel was then filled with a deoxygenated solution of $15 \%$ BMA in methanol and irradiated using the same conditions. Both photografting steps were followed by a thorough rinse with methanol.

The channels were finally filled with the degassed polymerization solution, which consisted of $24 \mathrm{wt} \%$ BMA, $16 \mathrm{wt} \%$ EDMA, $60 \mathrm{wt} \%$ 1-decanol and $0.4 \mathrm{wt} \%$ DMPA. A customized photo-mask was then used for UV irradiation $\left(2 \mathrm{~J} / \mathrm{cm}^{2}\right.$ at $\left.254 \mathrm{~nm}\right)$ in order to prepared a 1cm-long polymer monolith within the channel. The resulting monolith was thoroughly washed with methanol and dried with nitrogen. To confirm the integrity of the resulting monolith and its attachment to the channel walls, scanning electron micrographs of the monolith cross-section were taken using a Zeiss Evo LS15 Scanning Electron Microscope (SEM). For this purpose, the chip was scored across the channel using a scalpel, immersed in liquid nitrogen and then broken along the score mark in order to examine the channel crosssection. Prior to SEM measurements, the monolith was sputtered with gold using a Sputter coater (SC7640-Quorum Technologies).

\section{RESULTS AND DISCUSSION}

\section{Tensile Testing of Bond Strength}

Tensile testing of bonded blank samples (i.e., with no channels) was carried out in order to determine the quality and strength of the bond at the interface of two layers when the time of exposure to cyclohexane and the compression time were varied. In the first set of experiments, a single COC layer was exposed to cyclohexane vapor for $30 \mathrm{~s}$, $40 \mathrm{~s}$, or $50 \mathrm{~s}$; and then immediately brought into contact with a non-treated COC layer. Both layers were then compressed for 3, 4, or 5 min using a pneumatic press capable of applying a constant pressure of $0.4 \mathrm{MPa}$. UTS values obtained for the samples bonded following exposure to cyclohexane for $40 \mathrm{~s}(216 \mathrm{~Pa} \leq \mathrm{UTS} \leq 383 \mathrm{~Pa})$ were significantly higher than those obtained for a $30 \mathrm{~s}$ ( $150 \mathrm{~Pa} \leq \mathrm{UTS} \leq 283 \mathrm{~Pa})$ exposure. No conclusive UTS values could be calculated for samples exposed to cyclohexane for $50 \mathrm{~s}$ since measurements performed at the different compression times were not very reproducible. Figure 3 shows the stress-strain curves with the highest UTS values obtained for samples exposed to cyclohexane for $40 \mathrm{~s}$ at different compression times.

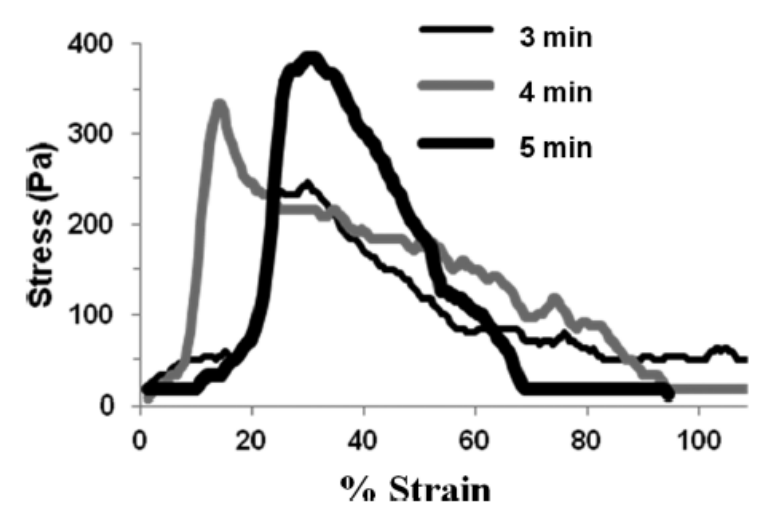

Figure 3.-Stress-strain curves obtained at different compression times following exposure of one layer to cyclohexane for $40 \mathrm{~s}$. 
Increased compression time resulted in an increase in the bonding strength. The same set of conditions for time of exposure to solvent vapor and compression were also applied during the fabrication of three-layer microfluidic chips integrating a single straight channel. Samples having been exposed to cyclohexane for $30 \mathrm{~s}$ showed weak bonding around the channel edges, which caused flow leakage in $90 \%(n=10)$ of the samples tested.

This was also observed in a small fraction of the samples prepared following an exposure time of $40 \mathrm{~s}$. Additionally, a significant number of samples previously exposed for $50 \mathrm{~s}$ presented blockage of the channel, most probably due to overexposure to solvent. Therefore, with the intent to improve the quality of the bonding around the channels, the two layers to be bonded were exposed to the solvent vapor before alignment and compression. Preliminary results actually showed better adhesion of layers around the channels. Thus, a new series of tensile testing experiments were performed following solvent exposure for the two layers to be bonded.

For the second set of experiments, a vice was used for compression instead of the pneumatic press, allowing a constant pressure of $0.9 \mathrm{MPa}$. COC films were again exposed to $30 \mathrm{~s}, 40 \mathrm{~s}$, and $50 \mathrm{~s}$, followed by 3, 4, or 5 min compression. Figure 4 illustrates the stressstrain curves with the highest UTS values obtained at different compression times for samples exposed for $30 \mathrm{~s}$ to the cyclohexane vapor. It is clear from comparison of Fig. 4 to Fig. 3 that the exposure of the two layers before bonding, instead of just one layer, as well as the increase in pressure during compression, resulted in a much stronger bonding at the interface.

Results of tensile tests for a bonding where the two layers were exposed for $30 \mathrm{~s}$ presented a maximum UTS value of $500 \mathrm{~Pa}$ at 5 min compression time, while the best result obtained when only one layer was exposed presented a maximum UTS value of $383 \mathrm{~Pa}$ at $40 \mathrm{~s}$ exposure and $5 \mathrm{~min}$ compression. This could be explained by the higher thickness of the diffusion interface when two layers are exposed as compared to exposure of only one of the layers. Longer compression times tended to lead to stronger bonding. For a significant number of samples, the bonding was so strong that they could not be completely delaminated, leading to sample fracture along the axis perpendicular to the applied stress before the tensile test was completed. Sample tearing mainly occurred for samples exposed to cyclohexane for $40 \mathrm{~s}$ and $50 \mathrm{~s}$. Slight misalignment in sample test set-up was also found to increase the changes of tearing perpendicular to the test direction as a result of increased stress concentration.

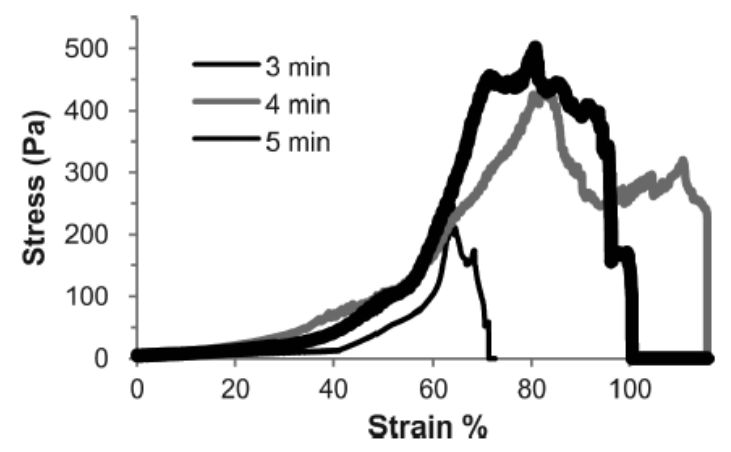

FIGURE 4.-Stress-strain curves obtained at different compression times following exposure of two layers to cyclohexane for $30 \mathrm{~s}$. 
It should be noted that all the samples exposed to the same conditions ( $n \geq 3$ ) showed similar behavior, confirming the conclusions deduced above.

Although samples exposed to $50 \mathrm{~s}$ presented a strong bonding, when the same bonding conditions were applied to the fabrication of three-layer single-channel microfluidic chips, most samples suffered again from channel clogging due to over exposure to cyclohexane. These results demonstrated that while it is a disadvantage to apply long exposure time as the material softens increasing the likelihood of channel blocking, much stronger bonding was achieved for exposures longer than $30 \mathrm{~s}$. Thus, it was concluded that for the purpose of the fabrication of microfluidic chips, an optimal bonding is obtained when the two layers to be bonded are exposed for $40 \mathrm{~s}$ to the solvent vapor, followed by compression at $0.9 \mathrm{MPa}$ for at least $4 \mathrm{~min}$. Three-layer microfluidic chips fabricated following this protocol presented a strong and uniform bonding with blockage-free channels

\section{Back Pressure Measurements}

In-channel back pressure measurements were carried out in microfluidic chips while pumping dyed DI water. As shown in Fig. 5, the maximum back pressure recorded for this test was $23 \mathrm{MPa}$ which was generated at a flow rate of $8 \mathrm{~mL} / \mathrm{min}$. No leakage was observed for this device at the flow rates tested. This illustrates the suitability of such platforms for high pressure applications. Moreover, for this method of COC bonding channel preparation, the recoded back pressure values from this work were among the highest reported to date. The average back pressure from five repeated tests was $14 \mathrm{MPa}$ which was obtained by averaging the maximum back pressure values obtained for different chips. When leakage was observed for some of these samples during the back pressure measurements, this occurred at the NanoPort connectors/ chip interface. Therefore, it is advised that special care should be taken in order to ensure a strong bonding of the connectors to the chip inlets/ outlets for high pressure applications.

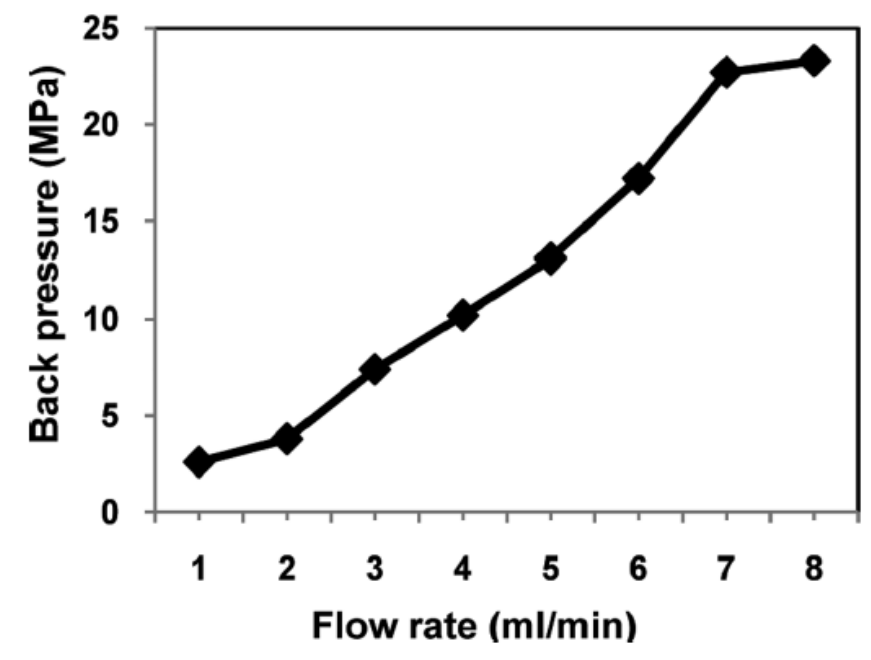

FIGURE 5.-Back pressure measurements at different flow rates. 


\section{Optical Transmission Measurements}

Figure 6 shows the average spectra recorded for three COC films before and after treatment following the protocol developed for solvent vapor bonding. No significant difference in optical transmission was observed between different periods of solvent exposure to cyclohexane for 30, 40, or $50 \mathrm{~s}$. These results confirmed the high optical transmission of COC films, with transmission values well above $90 \%$ along the wavelength range between $245 \mathrm{~nm}$ and $800 \mathrm{~nm}$, except for the range 260-280 $\mathrm{nm}$ where it gradually dropped down to $60 \%$. Similar behavior was observed for the treated COC films although the average transmission values were around $80 \%$, gradually dropping to approximately $30 \%$ at the $260-280 \mathrm{~nm}$ range. Therefore, these results demonstrate that the solvent vapor bonding method developed in this work is suitable for fabrication of microfluidic chips with high optical transmission in the visible region, allowing applications where on-chip optical detection is required. Furthermore, optimal transmission at the UV wavelengths typically used for in situ preparation of polymer monoliths in microchannels was also achieved, as demonstrated in Section 3.5.

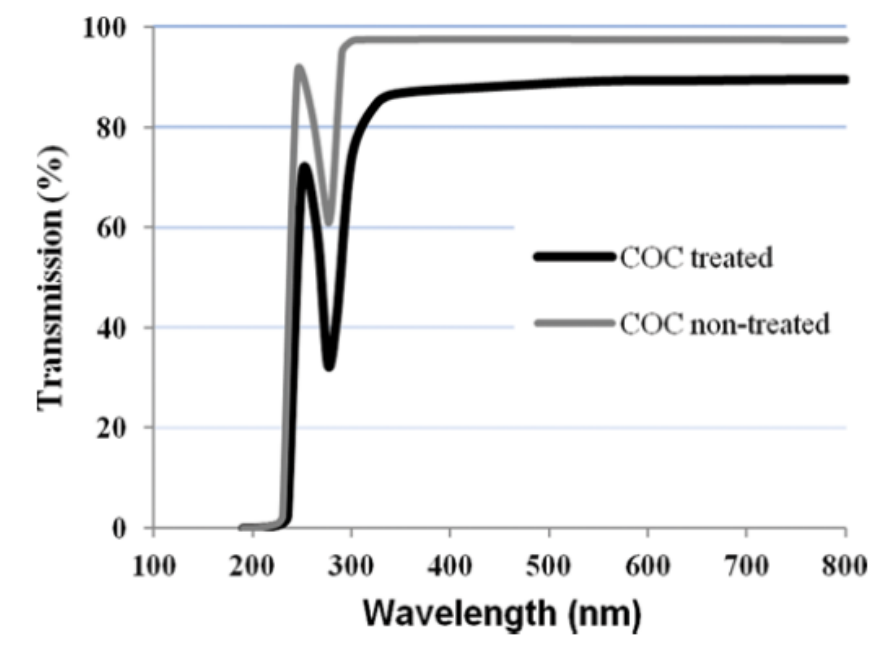

Figure 6.-Optical transmission spectra recorded for COC films before (nontreated) and after (treated) exposure to cyclohexane, UV irradiation, and compression.

\section{Micro-Mixers Evaluation}

Almost every chemical assay requires mixing of reagents with a sample. Micro-mixers are also used as a tool for dispersing immiscible liquids and forming micro-droplets [25], or as a separator for particles based on their different diffusion coefficients [26]. Micro-mixers are classified into two types, active and passive mixers. Passive micro-mixers are more widely used due to their simpler design, which is based on the principles of diffusion or natural/chaotic advection. Active mixers are more complex and expensive to fabricate. They generally use an external force which requires additional components. Since the first Tshaped micro-mixers, various designs have emerged in an effort to increase the contact surface between the fluids to be mixed, leading to an improvement in the molecular diffusion rate. Chaotic advection can be also enhanced by disrupting the laminar flow and creating 
transverse flows in the microchannel via introduction of obstacles in the flow path [27]. The resulting flow pattern shortens the diffusion path and thus improves mixing.

In order to evaluate the performance of the fabricated micro-mixers, an aqueous solution of Bromocresol purple or Bromothymol blue was pump into an empty channel through one of the channel inlets while pumping a $1 \mathrm{M} \mathrm{HCl}$ solution through the second inlet. The distance from the solutions contact point (i.e., T-junction) to the full mixing point (i.e., color change) was then measured. While the two solutions needed to travel $7-8 \mathrm{~mm}$ from the T-junction to mix in a classic T-shape mixer, mixing occurred within the first $2-3 \mathrm{~mm}$ from the T-junction in the other two types of mixers. As expected, these results showed that introducing obstacles and zigzag shaped turns into a straight $\mathrm{T}$-shaped channel improves the efficiency of mixing by promoting chaotic advection. Furthermore, no leaks were observed during the mixing tests, as shown in Fig. 7, confirming that the three COC layers used for fabrication of the micro-mixer exhibited an optimal and uniform bonding.

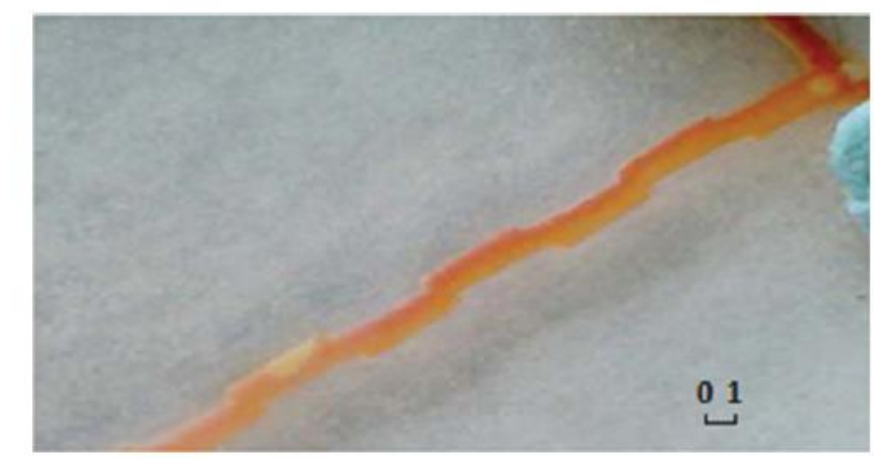

FIGURE 7.-Picture of a T-shaped mixer with obstacles on the wall during mixing tests. Scale bar indicates $1 \mathrm{~mm}$.

\section{Porous Polymer Monolith Polymerization}

Since the first fabrication of a polymer monolithic column inside a microfluidic channel [28], monolithic materials have been widely used in microfluidic platforms as separation columns, electrospray emitters, micro-valves, microreactors, and even micro-mixers [24]. UV-initiated polymerization of porous polymer monoliths has actually become a rather popular approach for integration of monoliths within microchannels. This method allows preparation of monoliths in specific areas of the microchannel by simply using a customized photomask. However, high transmission of the microfluidic chip layers in the UV region is required for such application. Thus, in situ preparation of a polymer monolith by UV-initiated polymerization was carried out in a three-layer microfluidic chip to actually demonstrate the optimal transmission properties of platforms fabricated following the method presented herein.

The resulting polymer monolith exhibited a uniform distribution along the channel length with no apparent voids and very sharp edges. Furthermore, excellent adhesion between the three COC layers bonded for fabrication of the chip could be observed. A closer look at the monolith cross section in Fig. 8 revealed the globular structure of the monolith and its good 
attachment to the channel walls, minimizing the risk of monolith displacement while pumping a fluid through the channel.

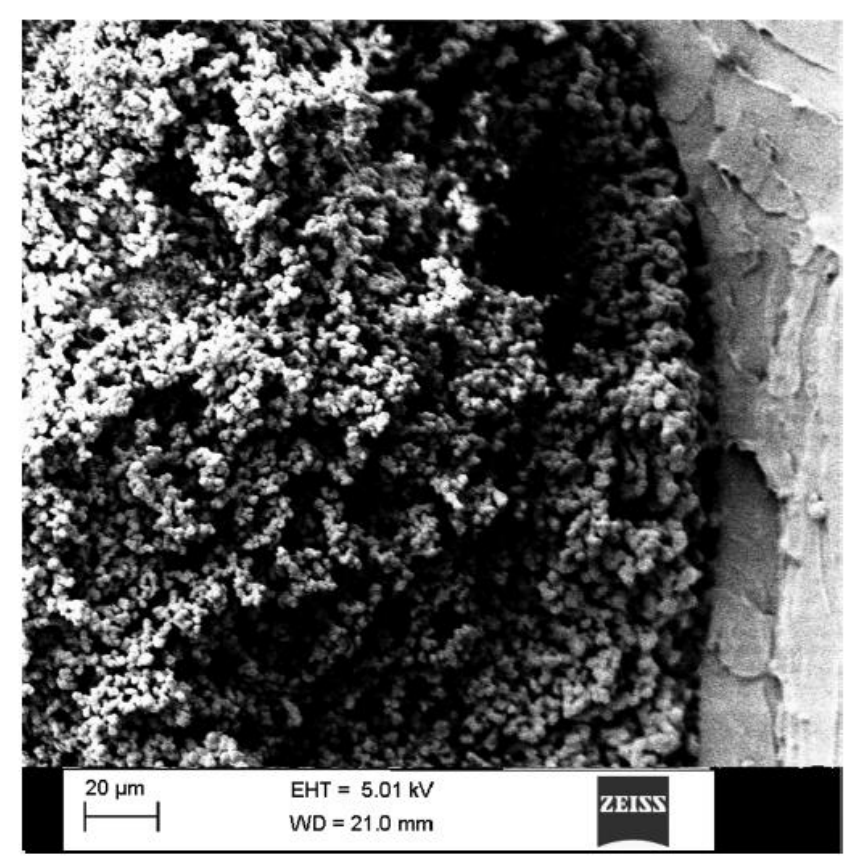

FIGURE 8.-SEM image of the monolith at the interface with the channel wall.

\section{CONCLUSION}

A new fabrication method for rapid production of polymeric multilayer microfluidic devices was developed and optimized. Three-layer microfluidic chips fabricated in less than an hour following this protocol presented a strong and uniform bonding with blockage-free channels, as illustrated by testing mixing capabilities in different micro-mixer designs. The resulting microfluidic devices also proved to be suitable for fabrication of polymer monolithic columns within the channels by in situ UV-initiated polymerization. This demonstrated that the optical transmission properties of COC films after solvent vapor bonding were still advantageous for such application. Moreover, these microfluidic devices were found to be able to withstand back pressures as high as $23 \mathrm{MPa}$, which makes them very attractive for high pressure applications in chemical synthesis, catalysis, and separation science. Thus, this work opens up new possibilities in low-cost and fast fabrication of microfluidic platforms with integration of several analytical operations (e.g., mixing, separation, detection) by using low-cost, fast prototyping methods. 


\section{REFERENCES}

1. Guu, Y.H.; Hocheng, H.; Chang, C.H. Study of piezoelectrically actuated micropumps with multiple parallel chambers. Materials and Manufacturing Processes 2008, 23(2), 210-215.

2. Mark, D.; Haeberle, S.; Roth, G.; von Stetten, F.; Zengerle, R. Microfluidic lab-on-a-chip platforms: requirements, characteristics and applications. Chemical Society Reviews 2010, 39(3), 1153-1182.

3. Becker, H.; Gaertner, C. Microfluidics an essential tool for product development in the life sciences. Chimica Oggi-Chemistry Today 2011, 29(4), 11-14.

4. Vázquez, M.; Brabazon, D.; Shang, F.; Omamogho, J.O.; Glennon, J.D.; Paull, B. Centrifugally-driven sample extraction, preconcentration and purification in microfluidic compact discs. Trac-Trends in Analytical Chemistry 2011, 30(10), 1575-1586.

5. Roy, S.; Yue, C.Y.; Venkatraman, S.S.; Ma, L.L. Low-temperature (below T-g) thermal bonding of COC microfluidic devices using UV photografted HEMA-modified substrates: high strength, stable hydrophilic, biocompatible surfaces. Journal of Materials Chemistry 2011, 21(38), 15031-15040.

6. Tsao, C.; DeVoe, D.L. Bonding of thermoplastic polymer microfluidics. Microfluidics and Nanofluidics 2009, 6(1), 1-16.Zhang, Z.; Luo, Y.; Wang, X.; Zheng, Y.; Zhang, Y.; Wang, L. Thermal assisted ultrasonic bonding of multilayer polymer

7. microfluidic devices. Journal of Micromechanics and Microengineering 2010, 20(1), 015036.

8. Ding, L.; Deng, Y.; Wang, D.; Yu, X. Fabrication and detection of microvalves for SPR array sensing. Microelectronic Engineering 2012, 91, 75-81.

9. Luo, Y.; Zhang, Z.; Wang, X.; Zheng, Y. Ultrasonic bonding for thermoplastic microfluidic devices without energy director. Microelectronic Engineering 2010, 87(11), 2429-2436.

10. Piruska, A.; Nikcevic, I.; Lee, S.; Ahn, C.; Heineman, W.; Limbach, P.; Seliskar, C. The autofluorescence of plastic materials and chips measured under laser irradiation. Lab on $a$ Chip 2005, 5(12), 1348-1354.

11. Becker, H.; Gartner, C. Microfluidics and the life sciences. Science Progress 2012, 95( Pt 2), 175-198.

12. Wu, J.; Gu, M. Microfluidic sensing: state of the art fabrication and detection techniques. Journal of Biomedical Optics 2011, 16(8), 080901.

13. Nunes, P.S.; Ohlsson, P.D.; Ordeig, O.; Kutter, J.P. Cyclic olefin polymers: emerging materials for lab-on-a-chip applications. Microfluidics and Nanofluidics 2010, $9(2-3)$, $145-161$.

14. Tsao, C.W.; Hromada, L.; Liu, J.; Kumar, P.; DeVoe, D.L. Low temperature bonding of PMMA and COC microfluidic substrates using UV/ozone surface treatment. Lab on a Chip 2007, 7(4), 499-505. 
15. Ogilvie, I.R.G.; Sieben, V.J.; Floquet, C.F.A.; Zmijan, R.; Mowlem, M.C.; Morgan, H. Reduction of surface roughness for optical quality microfluidic devices in PMMA and COC. Journal of Micromechanics and Microengineering 2010, 20(6), 065016.

16. Gaspar, A.; Salgado, M.; Stevens, S.; Gomez, F.A. Microfluidic "thin chips" for chemical separations. Electrophoresis 2010, 31(15), 2520-2525.

17. O'Toole, M.; Lau, K.T.; Diamond, D. Photometric detection in flow analysis systems using integrated PEDDs. Talanta 2005, 66(5), 1340-1344.

18. Mair, D.A.; Rolandi, M.; Snauko, M.; Noroski, R.; Svec, F.; Frechet, J.M.J. Roomtemperature bonding for plastic high-pressure microfluidic chips. Analytical Chemistry 2007, 79(13), 5097-5102.

19. Jena, R.K.; Yue, C.Y. Cyclic olefin copolymer based microfluidic devices for biochip applications: ultraviolet surface grafting using 2-methacryloyloxyethyl phosphorylcholine. Biomicrofluidics 2012, 6(1), 012822.

20. Cherdron, H.; Brekner, M.; Osan, F. Cycloolefin copolymers-a new class of transparent thermoplastic polymers. Angewandte Makromolekulare Chemie 1994, 223, 121-133.

21. Chen, G.; Svec, F.; Knapp, D.R. Light-actuated high pressure-resisting microvalve for onchip flow control based on thermo-responsive nanostructured polymer. Lab on a Chip 2008, 8(7), 1198-1204.

22. Mengeaud, V.; Josserand, J.; Girault, H.H. Mixing processes in a zigzag microchannel: finite element simulations and optical study. Analytical Chemistry 2002, 74(16), 42794286.

23. Wong, S.H.; Bryant, P.; Ward, M.; Wharton, C. Investigation of mixing in a cross-shaped micromixer with static mixing elements for reaction kinetics studies. Sensors and Actuators B: Chemical 2003, 95(1 -3), 414-424.

24. Vázquez, M.; Paull, B. Review on recent and advanced applications of monoliths and related porous polymer gels in micro-fluidic devices. Analytica Chimica Acta 2010, $668(2), 100-113$.

25. Haverkamp, V.; Ehrfeld, W.; Gebauer, K.; Hessel, V.; Lowe, H.; Richter, T.; Wille, C. The potential of micromixers for contacting of disperse liquid phases. Fresenius Journal of Analytical Chemistry 1999, 364(7), 617-624.

26. Weigl, B.H.; Yager, P. Microfluidics-microfluidic diffusion-based separation and detection. Science 1999, 283(5400), 346-347.

27. Stroock, A.; Dertinger, S.; Ajdari, A.; Mezic, I.; Stone, H.; Whitesides, G. Chaotic mixer for microchannels. Science 2002, 295(5555), 647-651.

28. Ericson, C.; Holm, J.; Ericson, T.; Hjerten, S. Electroosmosis- and pressure-driven chromatography in chips using continuous beds. Analytical Chemistry 2000, 72(1) , 8187. 